\title{
Global Franck-Condon Breakdown Resulting from Cooper Minima
}

\author{
R. M. Rao ${ }^{1}$ and E. D. Poliakoff ${ }^{1,2}$ \\ ${ }^{1}$ Department of Physics and Astronomy, Louisiana State University, Baton Rouge, Louisiana 70803 \\ ${ }^{2}$ Department of Chemistry, Louisiana State University, Baton Rouge, Louisiana 70803 \\ Kwanghsi Wang and V. McKoy \\ Arthur Amos Noyes Laboratory of Chemical Physics, California Institute of Technology, Pasadena, California 91125
}

(Received 1 December 1995)

\begin{abstract}
Using $\mathrm{N}_{2} 2 \sigma_{u}^{-1}$ photoionization as an example, we present the first measurements and calculations of photoion vibrational distributions for an extended energy range $\left(5 \leq E_{k} \leq 230 \mathrm{eV}\right)$. The results show a striking breakdown of the Franck-Condon approximation over a $100 \mathrm{eV}$ range. We show that this coupling between vibrational and electronic motion arises from a dependence of Cooper minima on molecular bond length. Based on this mechanism, the effect is expected to be common for molecular systems.
\end{abstract}

PACS numbers: 33.80.Eh, 31.15.-p, 33.50.Dq

The energy dependence of vibrationally resolved partial cross sections is central in the interpretation of molecular photoionization and detachment data. A clear picture of vibrational aspects of photoionization is a prerequisite for interpreting intensities quantitatively [1], and consequently for extracting accurate ionic potential surfaces. As examples, note that vibrationally resolved photoelectron spectra are essential ingredients for probing the chemical transition state [2] and extracting the structure of atomic and molecular clusters [3]. In interpretations of photoionization and photodetachment spectra, it is usually assumed that the vibrational intensities are governed by the Franck-Condon (FC) approximation, and hence that the ratio of cross sections for alternative vibrational levels is constant with photon energy. In this Letter, we report the first observation of coupling between electronic and vibrational motion which is global in extent, extending over a range of more than $100 \mathrm{eV}$. With $\mathrm{N}_{2} 2 \sigma_{u}^{-1}$ photoionization as an example, we show that the source of this Franck-Condon breakdown is a strong dependence of Cooper minima on internuclear separation over a broad energy range. Moreover, because of the underlying dynamics of the breakdown, this behavior is expected to be the rule rather than the exception in molecular photoionization. Viewed in a larger context, these results provide fundamental insights into the correlation between nuclear and electronic degrees of freedom.

Cooper minima [4,5] are ubiquitous for atomic and molecular systems. They originate from a change in sign of the dipole matrix element as a function of photon energy, so that the matrix element goes through zero. For molecular systems, the effects of Cooper minima on photoelectron angular distributions are well documented [5]. However, the influence of cooper minima on molecular vibration over a wide spectral range has never been explored [6,7], and that is the topic of the current study. We show-both experimentally and theoretically-that the $\mathrm{N}_{2} 2 \sigma_{u}^{-1}$ Cooper minima are strongly $R$ dependent, and that an important manifestation of this $R$ dependence is a coupling between electronic and nuclear motion. This study demonstrates that Cooper minima can result in deviations from Franck-Condon behavior over a broader range than any previously studied mechanism (by more than an order of magnitude). In so doing, this study highlights the molecular character of the photoelectron dynamics, even for scattering energies well in excess of those normally deemed relevant (i.e., energies on the order of a chemical bond, or approximately $4 \mathrm{eV}$ ).

Our experiment accesses vibrationally resolved photoionization data over a wide spectral range by exploiting dispersed fluorescence from excited photoions [8]. Gasphase nitrogen molecules are ionized by monochromatized synchrotron radiation, and the fluorescence from the excited photoions is collected, dispersed, and detected:

$$
\begin{aligned}
\mathrm{N}_{2}\left(X^{1} \Sigma_{g}^{+}, v_{0}=0\right)+h \nu_{\mathrm{exc}} \rightarrow & \mathrm{N}_{2}^{+}\left(B^{2} \Sigma_{u}^{+}, v^{+}\right)+e^{-} \\
& \downarrow \\
& \mathrm{N}_{2}^{+}\left(X^{2} \Sigma_{g}^{+}, v^{\prime \prime}\right) \\
& +h \nu_{v^{+}} v^{\prime \prime}
\end{aligned}
$$

The strategy is that the fluorescence intensity originating from a particular vibrational level is proportional to the partial photoionization cross section for that level [9]. An important feature of this method is that the fluorescence bandwidth is uncoupled from the excitation bandwidth, thereby enabling us to obtain highly resolved data on the photoions while simultaneously exploiting the broad tunability of synchrotron radiation [10]. Synchrotron radiation from the Louisiana State University Center for Advanced Microstructures and Devices (CAMD) facility is monochromatized $\left(\Delta h \nu_{\mathrm{exc}} \approx 0.5 \mathrm{eV}\right)$ by a 6 -m plane grating monochromator [11] and used to ionize the target molecules. The $\mathrm{N}_{2}$ gas is introduced into the interaction region via a free-jet supersonic expansion. Data are 
obtained over a range of stagnation pressures to test for secondary processes. The results are free of artifacts and are reproducible at pressures below $10^{-4}$ Torr. A complete discussion of the experiment will be given elsewhere [12].

The computational procedure used here has been discussed in detail previously [13]. To obtain the photoelectron orbitals, we use an iterative procedure based on the Schwinger variational principle to solve the LippmannSchwinger equation [13]. Calculations are performed at the Hartree-Fock frozen-core level, which is sufficient to characterize the key dynamical aspects. The ground state wave function of $\mathrm{N}_{2}$ is obtained at the self-consistent-field level [12]. A single-center expansion around the center of mass shows the $2 \sigma_{u}$ orbital to have $90.65 \% p, 6.66 \%$ $f$, and $1.52 \% h$ character at the equilibrium internuclear distance of $R_{e}=2.0743$ a.u. This orbital composition changes slowly from $96.86 \% p$ and $2.62 \% f$ character at an internuclear distance of 1.25 a.u. to $81.25 \% p$ and $11.30 \% f$ character at $R=3.5$ a.u. Note that the calculations assume the validity of the Born-Oppenheimer approximation $[12,13]$, i.e., the electronic wave function depends parametrically on bond distance. Thus, the observation of nonconstant vibrational branching ratios reflects this parametric, rather than dynamic, electronicnuclear coupling.

Figure 1 shows the portion of the fluorescence spectrum for $\mathrm{N}_{2}^{+}\left(B^{2} \Sigma_{u}^{+}, v^{+} \rightarrow X^{2} \Sigma_{g}^{+}, v^{\prime \prime}\right)$ which includes transitions originating from the $v^{+}=0,1$, and 2 levels. Vibrational branching ratios are extracted from the intensity ratios, as described elsewhere [9]. Spectra are obtained for photon energies from $25 \leq h \nu_{\text {exc }} \leq 250 \mathrm{eV}$, and the derived vibrational branching ratios are plotted as a function of excitation photon energy in Fig. 2. (The I.P. for $2 \sigma_{u}^{-1}$ is $18.76 \mathrm{eV}$ [14].) The deviations from Franck-Condon behavior are significant in the $v^{+}=1 / v^{+}=0$ ratio. The FC breakdown is even more evident in the much weaker $v^{+}=2$ channel. This behav-

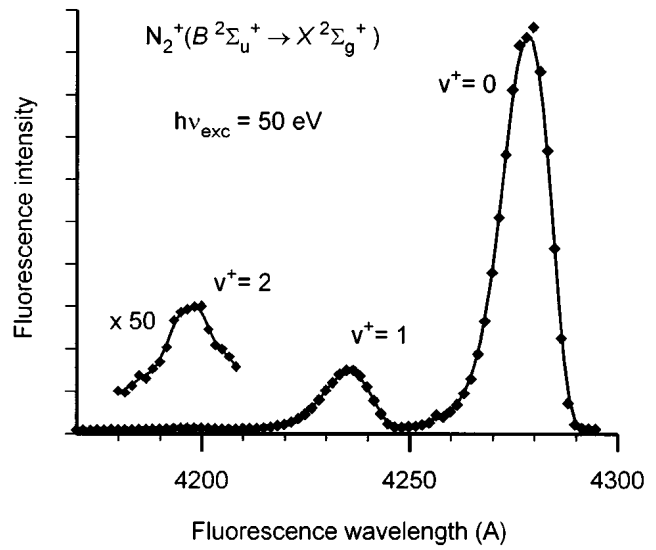

FIG. 1. Fluorescence spectrum from $\mathrm{N}_{2}^{+}$showing $B^{2} \Sigma_{u}^{+}$, $v^{+} \rightarrow X^{2} \Sigma_{g}^{+}, v^{\prime \prime}$ transitions. $v^{+}-v^{\prime \prime}=-1$ for transitions shown [14]. ior is expected as deviations from Franck-Condon behavior are usually enhanced for weaker channels [9]. While the absolute partial cross sections do not deviate substantially from Franck-Condon predictions, the branching ratios (which emphasize their relative magnitude) highlight the deviations clearly. The agreement between theory and experiment is quite good for the $v^{+}=1 / v^{+}=0$ results, while there is only qualitative agreement for the $v^{+}=2 / v^{+}=0$ results. It is not surprising that the agreement is not as good for the $v^{+}=2 / v^{+}=0$ curves, because the cross section is so weak that effects not included at the independent particle level can influence the ratios [15]. For comparison, the top frame of Fig. 2 shows the $v^{+}=1 / v^{+}=0$ curve for $3 \sigma_{g}^{-1} / \mathrm{N}_{2}$ photoionization on the same energy scale as the present $2 \sigma_{u}^{-1}$ results. The top frame exhibits the well-known $3 \sigma_{g} \rightarrow k \sigma_{u}$ shape resonance [16,17], which is usually considered to be very broad, but its width is dwarfed by the features in the $2 \sigma_{u}^{-1}$ curves. We emphasize two empirical aspects of the results, namely, the energy at which the excursion

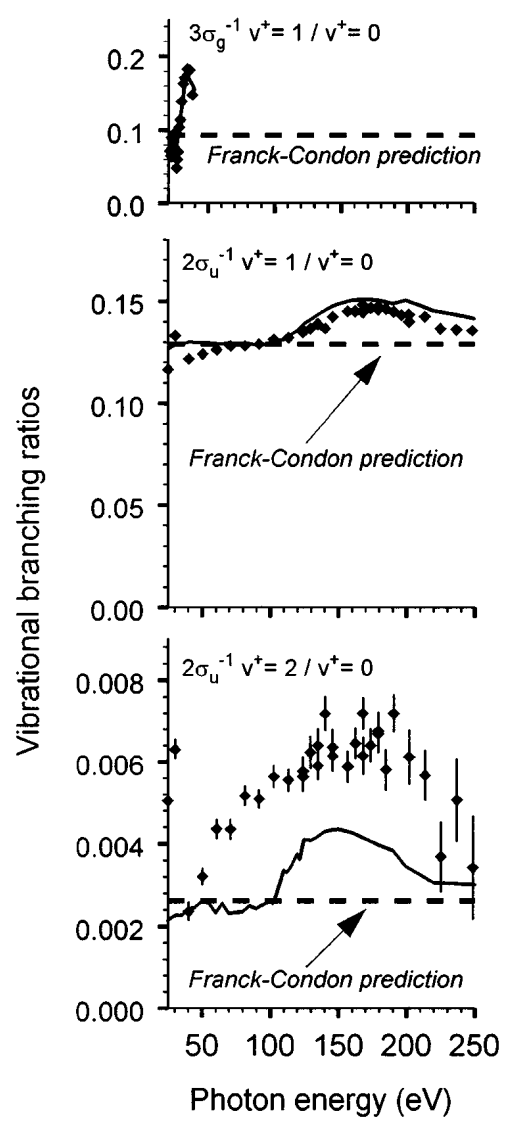

FIG. 2. Top frame: shape resonance induced FC breakdown (expt: Ref. [16], theory: Ref. [17]). Middle frame: $v^{+}=$ $1 / v^{+}=0$ ratio curve for $\mathrm{N}_{2} 2 \sigma_{u}^{-1}$ photoionization showing Cooper minimum-induced FC breakdown. Bottom frame: $v^{+}=2 / v^{+}=0$ ratio curve for $\mathrm{N}_{2} 2 \sigma_{u}^{-1}$ photoionization. The deviation from FC behavior is even more pronounced. All three frames have the same energy axis. Diamonds are experimental data and the solid lines are theoretical. 
occurs, and its range. First, the dominant deviations begin at $h \nu_{\mathrm{exc}} \approx 100 \mathrm{eV}$ and peaks at $160 \mathrm{eV}$, indicating that molecular aspects of the process (i.e., vibrational coupling) become pronounced at more than $80 \mathrm{eV}$ above the electron ejection threshold. It is usually implicit that molecular effects in such spectra are limited in extent in the continuum. The current results clearly belie this assumption. The second characteristic of the FC breakdown, the width of the feature, is important for interpretations of photoelectron spectra. When one tests for the validity of the FC approximation, it is generally assumed to be sufficient to check at photon energies separated by a few $\mathrm{eV}$, and to conclude that the FC approximation is valid if the relative vibrational intensities do not change substantially. The results shown in Fig. 2 demonstrate that changes in relative intensities can occur so gradually that normal tests for vibrational coupling are not sufficient. Experiments cannot be performed for all systems over such extended ranges, underscoring the importance of employing theory as a tool for interpreting experimental results.

To interpret the observed Franck-Condon breakdown, we note that there is a clue provided by a recent rotationally resolved study of $\mathrm{N}_{2} 2 \sigma_{u}^{-1}$ photoionization $[8,10]$, where we observed that the rotational branching ratios exhibit inflection points at $h \nu_{\mathrm{exc}} \approx 120 \mathrm{eV}$. Theoretical work showed that the energy dependence of these rotational branching ratios resulted from Cooper minima in the $l=2$ and $l=4$ partial waves of the $2 \sigma_{u} \rightarrow k \sigma_{g}$ channel. We therefore turn our attention to the dipole strengths for these components, which are shown in Fig. 3 for different values of $R$. Cooper minima are clearly visible for both these components. Moreover, the minima show a pronounced shift to lower photon energies with increasing internuclear separation. The peaks in the bottom two frames of Fig. 2 can be traced directly to the Cooper minima shifts in Fig. 3.

While we have explained the basis for the FranckCondon breakdown phenomenologically - as illustrated by Figs. 2 and 3 -it is still necessary to explain why the Cooper minima are shifting in the first place. There are two likely mechanisms which can contribute to the Cooper minima shifts, and both may play a role here. First, there is a gradual change of the angular momentum composition of the target molecular orbital with bond length. As a result, the Cooper minima are sampled to differing extents by alternative vibrational levels, and this can result in an $R$ dependence in the cross section curve [7]. Second, coupling of the angular momentum components of the molecular photoelectron orbital changes as the bond length changes. The first and second explanations can be characterized as initial and final state effects, respectively. Both effects may contribute to the observed behavior, and will be discussed in detail elsewhere [12].

Finally, it is helpful to describe other mechanisms for Franck-Condon breakdown in order to place the current study in context. Previous examples result from
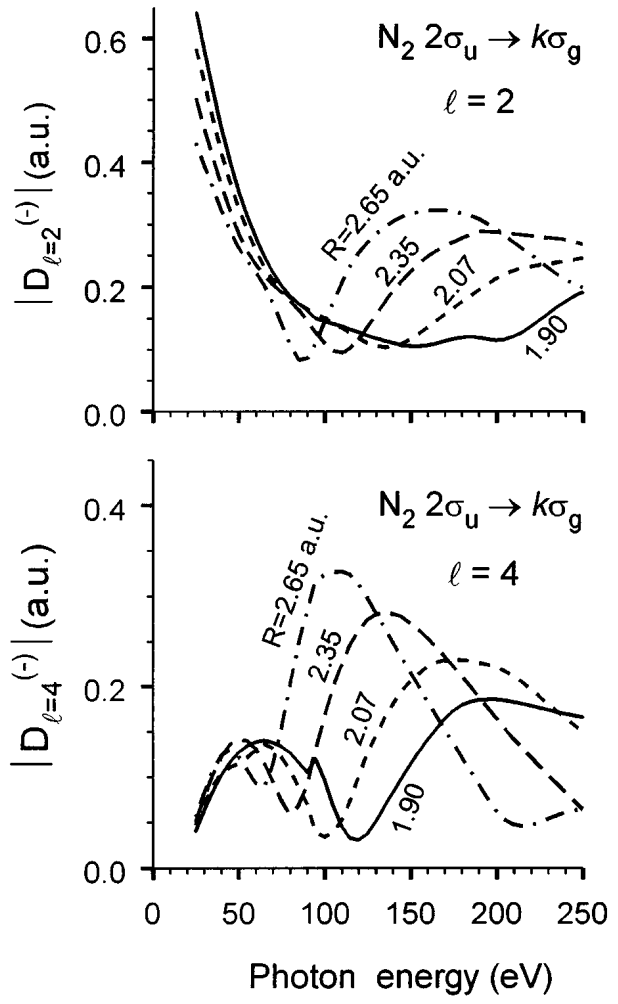

FIG. 3. Dipole strengths for the $\mathrm{N}_{2} 2 \sigma_{u} \rightarrow k \sigma_{g} l=2$ and $l=4$ partial waves. Note the $R$ dependence.

resonant excitation, either autoionization [18] or shape resonance $[16,17,19]$. In these cases, the eigenphase sum goes through $\pi$, and the cross section goes through an extremum. For the $\mathrm{N}_{2} 2 \sigma_{u}^{-1}$ channel, the cross section exhibits an inflection [12] rather than a local maximum or minimum. More importantly, the effect observed here is nonresonant, i.e., the eigenphase sum does not go through $\pi$ in the vicinity of the Cooper minimum [20]. We believe that this is unique, i.e., that a nonresonant mechanism is observed to cause coupling between electronic and vibrational degrees of freedom over a broad range.

The possibility of non-FC behavior due to a Cooper minimum has been recognized before [6,7]. In particular, Franck-Condon breakdown has been predicted for the pathological case of photoionization of hydrides in Rydberg target states [8]. The Franck-Condon breakdown predicted for hydrides is quite different from the effects observed in the current work. First, Rydberg states of hydrides are unusual in that an avoided crossing results in strong changes in the molecular orbital composition with internuclear separation. Stephens and co-workers [6] noted that this $R$ dependence could yield strong non-Franck-Condon effects in a resonance-enhanced multiphoton ionization and photoemission spectroscopy experiment. However, the non-Franck-Condon effects are limited to a narrow class of systems, and are also predicted to be limited in their spectral scope. 
In summary, we have observed Cooper minima induced Franck-Condon breakdown in vibrational branching ratios for $\mathrm{N}_{2} 2 \sigma_{u}^{-1}$ photoionization over an extremely broad range $\left(\Delta E_{\mathrm{exc}} \approx 100 \mathrm{eV}\right)$. This behavior is attributed to the $R$ dependence of the Cooper minima. Such effects are unexpected, and have the following implications. First, it is the broadest deviation from Franck-Condon behavior ever observed. Second, it is the first nonresonant mechanism which exhibits Franck-Condon breakdown. Third, it is expected to be phototypical for both simple and complex molecular systems. Fourth, very molecular aspects of photoionization are seen to be prevalent in a regime usually assumed to be dominated by atomiclike behavior. Fifth, it is a simple process which can be exploited for studying the correlation of electronic and nuclear motion. Indeed, the unifying theme provided by this and several previous studies $[8-10,15]$ is that photoionization opens many avenues for probing correlations between electronic and nuclear degrees of freedom. The present work demonstrates that the dynamics of Cooper minima provide a means of investigating electronic and vibrational correlations which are both accessible and useful.

We thank the CAMD staff for assistance. E.D.P. acknowledges support from the NSF (CHE-9315857) and the Louisiana LEQSF program. Work at Caltech was supported by the Air Force Office of Scientific Research, the Office of Health and Environmental Research of the U.S. Department of Energy, and the JPL/Caltech CRAY Y-MP2E/232 Supercomputer.

[1] J.H.D. Eland, Photoelectron Spectroscopy (Wiley, New York, 1974), p. 112.

[2] D. M. Neumark, Annu. Rev. Phys. Chem. 43, 153 (1992); R. B. Metz and D. M. Neumark, J. Chem. Phys. 97, 962 (1992).

[3] G.H. Lee, S. T. Arnold, J. G. Eaton, H. W. Sarkas, K. H. Bowen, C. Lugwigt, and H. Haberland, Z. Phys. 20, 9 (1991).
[4] J. W. Cooper, Phys. Rev. 128, 681 (1962); S. T. Manson, Phys. Rev. A 31, 3698 (1985).

[5] For a review of previous work on molecular Cooper minima, see T. A. Carlson, M. O. Krause, W. A. Svensson, P. Gerard, F. A. Grimm, T. A. Whitley, and B. P. Pullen, Z. Phys. D 2, 309 (1986).

[6] J. A. Stephens and V. McKoy, Phys. Rev. Lett. 62, 889 (1989).

[7] W. A. Chupka, J. Chem. Phys. 87, 1488 (1987).

[8] E. D. Poliakoff, H.C. Choi, R. M. Rao, A. G. Mihill, S. Kakar, K. Wang, and V. McKoy, J. Chem. Phys. 103, 1773 (1995), and references therein.

[9] L. A. Kelly, L. M. Duffy, B. Space, E. D. Poliakoff, P. Roy, S. H. Southworth, and M. G. White, J. Chem. Phys. 90, 1544 (1989).

[10] H. C. Choi, R. M. Rao, A.G. Mihill, S. Kakar, E. D. Poliakoff, K. Wang, and V. McKoy, Phys. Rev. Lett. 72, 44 (1994).

[11] E. Morikawa, J. D. Scott, E. D. Poliakoff, R. L. Stockbauer, and V. Saile, Rev. Sci. Instrum. 63, 1300 (1992).

[12] R. M. Rao, E. D. Poliakoff, K. Wang, and V. McKoy, (to be published).

[13] R. R. Lucchese, K. Tatsuka, and V. McKoy, Phys. Rep. 131, 147 (1986), and references therein.

[14] G. Herzberg, in Molecular Spectra and Molecular Structure (Krieger, Malabar, Florida, 1989), Vol. 1.

[15] S. Kakar, H.C. Choi, and E. D. Poliakoff, Chem. Phys. Lett. 190, 489 (1992).

[16] J.B. West, A. C. Parr, B.E. Cole, D. L. Ederer, R. Stockbauer, and J.L. Dehmer, J. Phys. B 13, L105 (1980).

[17] R. R. Lucchese and V. McKoy, J. Phys. B 14, L629 (1981).

[18] S. T. Pratt, P. M. Dehmer, and J. L. Dehmer, Chem. Phys. Lett. 105, 28 (1984).

[19] J. L. Dehmer, A. C. Parr, and S. H. Southworth, in Handbook on Synchrotron Radiation, edited by G. V. Marr (North-Holland, Amsterdam, 1987), Vol. II.

[20] M.S. Child, in Molecular Collision Theory (Academic, New York, 1975). 